\title{
Serum Gamma-Glutamyltransferase Levels Predict the Development of Cerebral Infarction in the Korean Population
}

\author{
Sang Min Lee (D) \\ Eunhee $\mathrm{Ha}^{2}$ \\ Jae-Hong Ryoo 3 \\ 'Department of Psychiatry, Kyung Hee \\ University Medical Center, Kyung Hee \\ University School of Medicine, Seoul, \\ Korea; ${ }^{2}$ Department of Occupational and \\ Environment Medicine, College of \\ Medicine, Ewha Womans University, \\ Seoul, Korea; ${ }^{3}$ Department of \\ Occupational and Environmental \\ Medicine, School of Medicine, Kyung Hee \\ University, Seoul, Korea
}

\begin{abstract}
Purpose: This study examined the relationship between serum gamma-glutamyltransferase concentration and the risk of cerebral infarction in the Koreans.

Materials and Methods: A total of 209,481 out of 223,551 participants in the National Health Information Database who received medical checkups in 2009 were included in the final analysis. The diagnosis code ICD I63 was used for identifying cerebral infarction cases and tracked the development of cerebral infarction by 2013. Cox proportional hazards model was used to calculate hazard ratios (HRs) for cerebral infarction and their confidence interval (CI).

Results: During a follow-up period of 915,387.5 person-years, 2403 incident cases of cerebral infarction developed between 2009 and 2013. After adjusting for multiple covariates, the hazard ratios (95\% confidence interval) for incident cerebral infarction, comparing the second, third, and fourth quartile of serum gamma-glutamyltransferase levels with the first quartile, were $1.11(0.98-1.27), 1.39$ (1.22-1.58), and 1.49 (1.29-1.71), respectively ( $P$ for trend $<0.001)$.
\end{abstract}

Conclusion: Elevated serum gamma-glutamyltransferase levels were independently associated with the future development of cerebral infarction in Koreans.

Keywords: gamma-glutamyltransferase, cerebral infarction, stroke

\section{Introduction}

Gamma-glutamyl transferase (GGT) is an enzyme localized on the external surface of the plasma membranes of various cells and tissues. ${ }^{1}$ GGT is a ubiquitous enzyme that catalyzes the hydrolysis of $\gamma$-glutamyl bonds in glutathione and glutamine, as well as the transfer of the released $\gamma$-glutamyl groups to amino acids or short peptides. GGT is involved in glutathione metabolism and plays an important role in antioxidant defense, detoxification, and inflammatory processes. ${ }^{2}$

GGT is the main enzyme that hydrolyzes the antioxidant glutathione. This hydrolysis reaction can increase the formation and development of atherosclerotic plaques by creating oxidation promoters. ${ }^{3}$ In second-generation enzymatic liver function tests, the GGT level was used as a sensitive enzymatic indicator of alcohol intake and many types of liver diseases. ${ }^{4}$ Many previous studies have considered GGT as a marker for alcohol consumption. ${ }^{5}$

Recently, serum GGT level was shown to be a predictive factor for cardiovascular diseases. ${ }^{6}$ Several studies have shown that atherosclerosis is associated with serum GGT levels, ${ }^{7,8}$ which suggests that GGT can be used as an early marker of
Correspondence: Jae-Hong Ryoo

Occupational and Medicine, Kyung Hee University, 23

Kyungheedae-ro, Dongdaemun-gu, Seoul, 02447, Korea

Tel +82-10-6235-2208

Fax +82-2-969-0792

Email armanil3I@naver.com 
atherosclerosis. ${ }^{9}$ Some studies have also shown that blood GGT activity is an independent biomarker of coronary artery calcification and that it is associated with cerebral infarctions. ${ }^{3,10,11}$ Studies with small sample sizes have shown a positive correlation between increased infarct area and elevated GGT levels in patients with acute ischemic strokes. ${ }^{12}$

Several epidemiological studies have shown that an elevated GGT level increases the risks of cardiovascular disease (CVD) and subsequent death. ${ }^{4,13}$ The Framingham offspring study was one of the first epidemiological studies to test the association between GGT levels and the risk of cardiovascular diseases. The study also revealed that GGT might predict the metabolic and cardiovascular risks associated with the onset of metabolic syndrome, incident CVD, and indicate whether the condition is fatal. ${ }^{13}$ However, GGT has not yet been used as a tool to predict the risk of strokes in clinical practice, for which more evidence is needed.

The National Health Insurance Corporation (NHIC) is a Korean national institution that provides the National Health Insurance Service (NHIS) to the Korean population. The NHIS data were used to establish the NHISNational Sample Cohort (NHIS-NSC) in 2002. ${ }^{14}$

In this study, we evaluated the risk of cerebral infarction based on GGT levels. An important objective was to assess the relationship GGT levels and cerebral infarction using retrospective cohort data of 209,481 Koreans from the NHIS-NSC database.

\section{Materials and Methods Data Sources}

The national health insurance system is used by almost the entire population (more than 97\%), which means that this database represents the use of medical services for all Koreans. ${ }^{14}$ Also, almost all Koreans over the age of 40 must have a medical checkup at least once every two years. NHIC in South Korea collects and stores information on all health checkups performed. Recently, after removing all information related to personal identification, the NHIS has provided the sample database for research purposes in the public interest. The incidence of cerebral infarction obtained by the National Statistical Office is linked information on health checkups included in the sample database provided by the NHIC.

This study protocol and analysis of the data was approved by the Institutional Review Board of Kyung Hee University Hospital (KHUH 2018-12-020). The institutional review board waived the requirement for informed consent because researchers accessed databases that were not identified retrospectively for analysis purposes.

\section{Study Participants}

A total of 223,551 participants who underwent medical health checkups in 2009 were included in the NHIS Database. Among them, 2387 individuals who had a cerebral infarction (ICD I63) from 2002 to 2009 at the time of medical checkup were excluded. Of the 221,164 participants, 11,683 were further excluded based on the following exclusion criteria that might influence cerebral infarction or GGT levels: 57 people did not have information about baseline GGT in 2009 and 11,626 previously had a diagnosis of cancer (ICD C00-C97) between 2002 and the time of the medical health examination in 2009. Because some participants met more than one exclusion criterion, the final subjects of analysis were 209,481, and they were observed the occurrence of cerebral infarction (Figure 1). The total follow-up period we calculated was 915,387.5 person-years, and the average follow-up period was identified as 4.37 (standard deviation [SD], 0.48) person-years.

\section{Health Survey Examinations and Laboratory Measurements}

The general health checkup conducted by the NHIC is carried out in two stages. A large-scale screening test is the first stage in determining the presence or absence of disease among ordinary people without symptoms. The second stage involves counseling for screening tests and more detailed testing to determine the presence of diseases. These medical health examinations include surveys of lifestyle and past medical history. Research data includes physical activities, anthropometric measurements, laboratory measurements, and information provided by questionnaires. Measurements of the amount of smoking was used on pack-years. Packyears measurements were calculated based on responses to smoking-related questions in the questionnaire. Consuming of $>3$ drinks per week was defined as alcohol use. The definition of being physically active was to engage in moderate-intensity physical activity for at least 30 minutes per day for at least 4 days a week or vigorous-intensity physical activity for at least 20 minutes a day for more than 4 days per week. The weight 


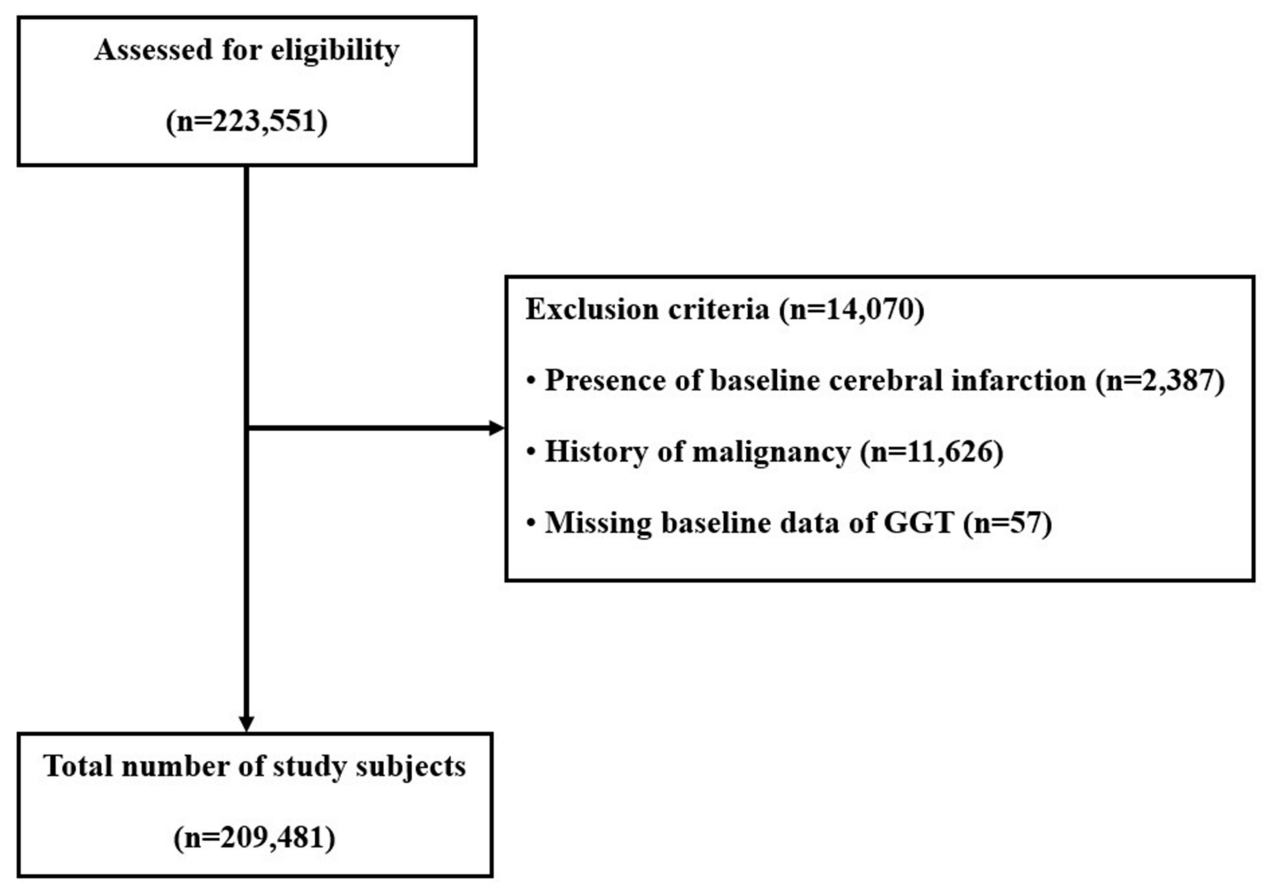

Figure I Flow chart of enrolled study subjects.

(kg) divided by the square of the height (m) was calculated for body mass index (BMI). Systolic and diastolic blood pressure (BP) were measured by experienced examiners. Laboratory data such as fasting blood glucose, total cholesterol, triglyceride, high-density lipoprotein cholesterol, low-density lipoprotein (LDL) cholesterol, serum creatinine ( $\mathrm{SCr}$ ), aspartate aminotransferase (AST), alanine aminotransferase, and GGT were measured by these participants under health examinations. The estimated glomerular filtration rate (eGFR) were calculated using the Chronic Kidney Disease Epidemiology Collaboration (CKD-EPI) equation to measure kidney function. ${ }^{15}$

\section{Outcome Definitions}

The NHIS database was merged in connection with the diagnosis of disease data from Statistics Korea. In this study, the start date was the first health checkup since 2009, and the last date of follow-up observation was the time of diagnosis of cerebral infarction until end of 2013. The ICD code I63 was used to diagnose cerebral infarction. The denoted with ICD code I63 was used for the diagnosis of cerebral infarction. The primary clinical endpoint in this study was a composite of the development of cerebral infarction.

\section{Statistical Analysis}

Data are expressed as mean \pm standard deviation or median (interquartile range) for continuous variables and as percentages of the number for categorical variables.

One-way analysis of variance and $\chi^{2}$-test were used to analyze the statistical differences among the characteristics of the study participants at the time of enrollment in relation to the quartile groups for baseline GGT levels.

The person-years were calculated as the sum of followup times from the baseline until the diagnosis of cerebral infarction development or until December 31, 2013.

To evaluate the associations between the quartile groups of baseline GGT levels and incident cerebral infarction, we used Cox proportional hazards models to estimate adjusted hazard ratios (HRs) and 95\% confidence intervals (CIs) for incident cerebral infarction by comparing the three highest quartiles of baseline GGT levels with the lowest quartile. The Cox proportional hazard models were adjusted for multiple confounding factors. In the multivariate models, this study included variables that could confuse the relationship between GGT and incident cerebral infarction, including age, sex, BMI, systolic BP, fasting blood glucose, total cholesterol, eGFR, smoking amount (pack-years), alcohol intake, and physical activity. We verified the proportional hazard assumption and tested the validity of the Cox proportional hazard models. 
The proportional hazard assumption was evaluated using the log-minus-log survival function, and graphically non-violations were found. A p-value less than $0.05(P<$ $0.05)$ is statistically significant. Statistical analyses were performed using the program SAS (Statistical Analysis Software 9.4, SAS Institute Inc, Cary, North Carolina, USA).

\section{Results}

During the 915,387.5 person-years of follow-up, 2403 $(1.15 \%)$ incident cases of cerebral infarction developed between 2009 and 2013. In Table 1, the baseline characteristics of the study participants related to the quartile groups at baseline GGT levels are presented. At the baseline of the study, the mean $( \pm \mathrm{SD})$ age of participants was $57.8( \pm 8.6)$ years and the BMI was $24.0( \pm 2.9) \mathrm{kg} / \mathrm{m}^{2}$. Significant differences were shown between all the listed variables and quartile groups of the baseline GGT levels.

Participants with incident cerebral infarction were older (67.7 vs 57.7 years) than those without incident cerebral infarction, and had relatively poor metabolic profile at baseline. As expected, significant statistical differences were identified in all clinical variables, except BMI, total cholesterol, LDL-cholesterol, SCr, and AST (Table 2).

Table 3 shows the HRs and 95\% CIs for cerebral infarction according to baseline GGT levels. In the unadjusted model, comparisons of incident cerebral infarction in quartiles 2, 3, and 4 vs quartile 1 (reference group) showed that the HRs and 95\% CIs were 1.26 (1.10$1.42), 1.52(1.35-1.72)$, and $1.54(1.36-1.73)$, respectively ( $P$ for trend $<0.001$ ).

These associations remained statistically significant even after further adjustments for covariates in the multivariate adjusted model. The adjusted HRs and 95\% CIs for incident cerebral infarction were $1.11(0.98-1.27), 1.39$ (1.22-1.58), and 1.49 (1.29-1.71), respectively ( $P$ for trend $<0.001)$.

\section{Discussion}

It has been confirmed through this study that serum GGT activity in both men and women was significantly correlated with the risk of cerebral infarction. The risk of cerebral infarction was also proportional to GGT levels, even after adjusting for stroke-related risk factors. Therefore, this study demonstrates that GGT, which has been known as a predictive factor for alcohol consumption, has potential usefulness as a risk factor for cerebral infarction.
It also suggests the possibility that GGT may play a role in reducing the incidence of cerebral infarction and related disease burdens from a public health perspective.

Some evidence have been identified for the relationship between GGT levels and the risk of stroke. The EUROSTROKE study have confirmed that high levels of GGT and increased risk of stroke are involved in three European cohorts. In this study, hemorrhagic stroke was more linked to GGT than cerebral infarction. ${ }^{16}$ In a cohort study of Korean subjects, serum GGT values are independently associated and are significant dose-response relationship with the risk of hemorrhagic stroke, not ischemic stroke or ischemic heart disease. ${ }^{17}$ However, there are another cohort study that have found that GGT is more consistently correlated with ischemic stroke. ${ }^{18}$ A Finnish study has confirmed that serum GGT was associated with the risk of ischemic stroke as well as overall stroke. The Finnish study showed the risk ratios for men and women in total stroke were 1.45 and 1.48 while ischemic stroke was 1.51 and 1.59 , respectively, over 7- or 12-year follow-up periods. ${ }^{11,19}$ The findings of the present study are valuable because it highlights the relationship between GGT levels and cerebral infarction, especially in case of ischemic stroke. Another cohort study found that GGT level in patients with acute ischemic stroke were correlated with all-cause or cardiovascular disease mortality. ${ }^{20}$

Based on recent findings, GGT has drawn attention for its close association with the occurrence of atrial fibrillation, one of the major risk factor for ischemic stroke. Elevated GGT levels induce atrial fibrillation, which has been shown to lead to stroke. Increased GGT levels may indirectly reflect the increased oxidative stress and inflammatory processes, which may be a risk factor for cardiac metabolism or predisposition to atrial fibrillation. ${ }^{21-25}$ Nevertheless, follow-up studies are needed to investigate whether and how mechanisms associated with GGT levels differ depending on the subtype of ischemic stroke.

The main strengths of this longitudinal cohort study are the use of national database from population-based registers and well-organized anthropometric and laboratory measurements. These researchers are therefore determined to have obtained reliable metabolic components and diagnostic information related to cerebral infarction. In addition, participants with diseases 


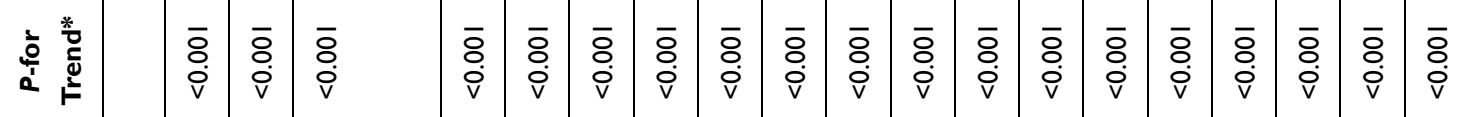

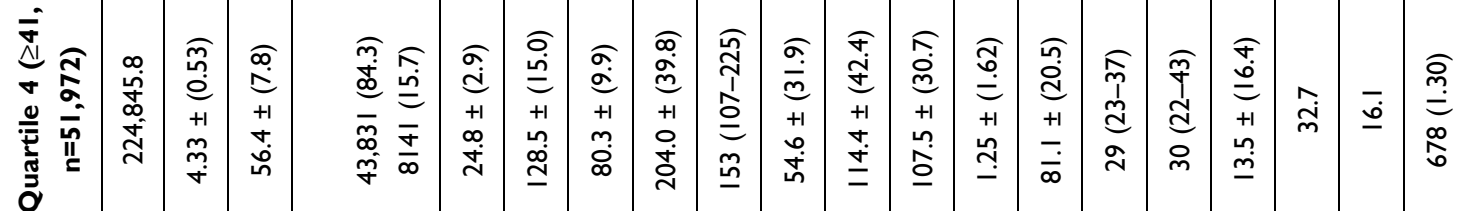

$\overline{\mathrm{v}}$

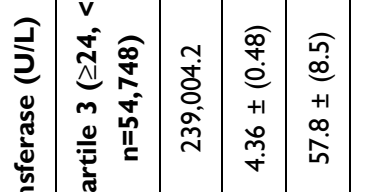

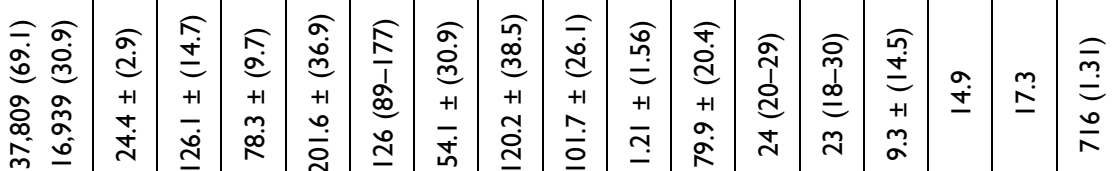

$\pm$

वैं

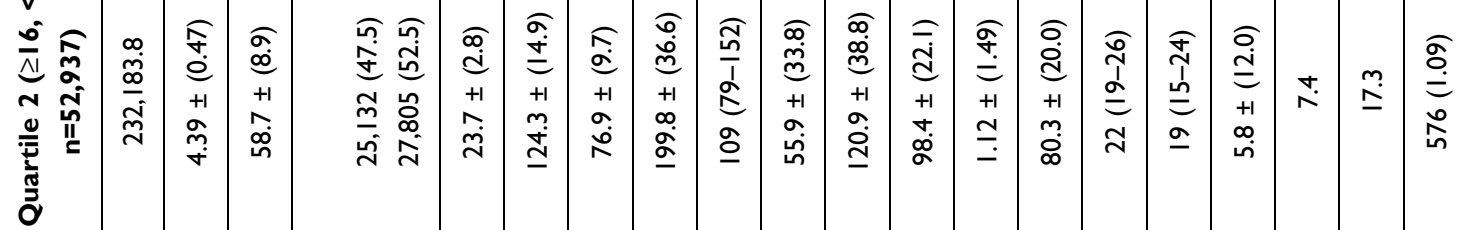

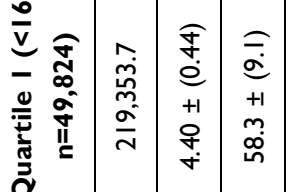

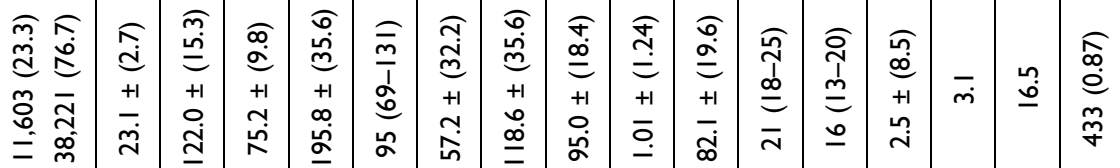

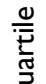

\section{=}


Table 2 Comparison Between Participants with and without Incident Cerebral Infarction

\begin{tabular}{|c|c|c|c|}
\hline Characteristic & $\begin{array}{l}\text { Without Incident Cerebral } \\
\text { Infarction }(\mathrm{N}=\mathbf{2 0 7 , 0 7 8 )}\end{array}$ & $\begin{array}{l}\text { With Incident Cerebral } \\
\text { Infarction }(\mathrm{N}=\mathbf{2 4 0 3})\end{array}$ & $P$-value* \\
\hline Age (years) & $57.7 \pm(8.6)$ & $66.7 \pm(9.4)$ & $<0.001$ \\
\hline Gender & & & $<0.001$ \\
\hline Male (\%) & I I6,882 (56.4) & $1493(62.1)$ & \\
\hline Female (\%) & $90,196(43.6)$ & $910(37.9)$ & \\
\hline BMI $\left(\mathrm{kg} / \mathrm{m}^{2}\right)$ & $24.0 \pm(2.9)$ & $24.1 \pm(2.9)$ & 0.188 \\
\hline Systolic BP (mmHg) & $125.2 \pm(15.1)$ & $131.9 \pm(17.3)$ & $<0.001$ \\
\hline Diastolic BP (mmHg) & $77.7 \pm(9.9)$ & $80.1 \pm(10.8)$ & $<0.001$ \\
\hline Total cholesterol (mg/dL) & $200.4 \pm(37.4)$ & $201.4 \pm(40.0)$ & 0.229 \\
\hline Triglyceride (mg/dL) & $142.0 \pm(94.3)$ & $154.9 \pm(97.8)$ & $<0.001$ \\
\hline HDL-cholesterol (mg/dL) & $55.4 \pm(32.3)$ & $52.6 \pm(29.8)$ & $<0.001$ \\
\hline LDL-cholesterol (mg/dL) & $118.5 \pm(39.0)$ & $119.2 \pm(39.9)$ & 0.401 \\
\hline Fasting blood glucose $(\mathrm{mg} / \mathrm{dL})$ & $100.6 \pm(25.1)$ & $108.7 \pm(35.5)$ & $<0.001$ \\
\hline $\mathrm{SCr}(\mathrm{mg} / \mathrm{dL})$ & $1.15 \pm(1.49)$ & $1.14 \pm(1.28)$ & 0.597 \\
\hline eGFR $\left(\mathrm{mL} / \mathrm{min}\right.$ per $\left.1.73 \mathrm{~m}^{2}\right)$ & $80.9 \pm(20.2)$ & $74.3 \pm(19.3)$ & $<0.001$ \\
\hline AST (U/L) & $26.5 \pm(16.3)$ & $27.0 \pm(24.4)$ & 0.421 \\
\hline ALT (U/L) & $25.4 \pm(19.2)$ & $24.4 \pm(22.2)$ & 0.025 \\
\hline GGT (U/L) & $39.1 \pm(53.9)$ & $43.8 \pm(60.7)$ & $<0.001$ \\
\hline Smoking amount (pack-year) & $7.8 \pm(13.8)$ & $10.3 \pm(16.6)$ & $<0.001$ \\
\hline Alcohol intake (\%) & 14.6 & 16.5 & 0.010 \\
\hline Physical activity (\%) & 16.8 & I5.I & 0.029 \\
\hline
\end{tabular}

Notes: Data are expressed as means (standard deviation) or percentages. $* P$-value by $t$-test for continuous variables and Chi square test for categorical variables.

that could affect cerebral infarction or GGT levels were excluded from the analysis. This process allowed us to quantify the robust effects of GGT on cerebral infarction. Another advantage of this study is that a single ICD code (I63) selected by the physician who performed the diagnosis was analyzed.

There are a number of limitations related to the analysis in this research. First, our results were based on raw data from medical examinations and related questionnaires; there may therefore be a collection bias. Second, the follow-up period of this study was 4.37 years, relatively short to confirm the long-term effects of GGT on cerebral infarction. Further research should be conducted to overcome these limitations.

\section{Conclusion}

These results indicated that increased GGT levels may be associated with the risk of cerebral infarction. This association was significant even after adjustment for multiple baseline covariates.

\section{Clinical Implication}

The results of this study show that a high level of GGT increase the risk of cerebral infarction, in addition to the previously known increased risk of alcohol intake. In addition to known association with alcohol consumption, GGT is meaningful as an indicator of oxidative damage and is clinically useful because it can be easily identified through simple routine blood chemistry in general clinical setting. 
Table 3 Hazard Ratios (HRs) and 95\% Confidence Intervals (Cl) for the Incidence of Cerebral Infarction According to the Quartile Groups of Serum GGT Levels

\begin{tabular}{|c|c|c|c|c|c|}
\hline & \multirow{2}{*}{$\begin{array}{l}\text { Person- } \\
\text { Year }\end{array}$} & \multirow{2}{*}{$\begin{array}{c}\text { Incidence } \\
\text { Cases }\end{array}$} & \multirow{2}{*}{$\begin{array}{c}\text { Incidence } \\
\text { Density } \\
\text { (per 10,000 } \\
\text { Person-Year) }\end{array}$} & \multicolumn{2}{|c|}{ HR (95\% Cl) } \\
\hline & & & & Unadjusted & Multivariate Adjusted Model* \\
\hline$\gamma$-glutamyltransferase & & & & & \\
\hline Quartile I & $219,353.7$ & 433 & 19.7 & 1.00 (reference) & 1.00 (reference) \\
\hline Quartile 2 & $232,183.8$ & 576 & 24.8 & $1.26(1.10-1.42)$ & I.II (0.98-I.27) \\
\hline Quartile 3 & $239,004.2$ & 716 & 29.9 & $1.52(1.35-1.72)$ & $1.39(1.22-1.58)$ \\
\hline Quartile 4 & $224,845.8$ & 678 & 30.1 & $1.54(1.36-1.73)$ & $1.49(|.29-| .7 I)$ \\
\hline$P$ for trend & & & & $<0.001$ & $<0.001$ \\
\hline Age & & & & & $1.10(1.10-1.11)$ \\
\hline Gender (female Vs male) & & & & & $0.78(0.70-0.86)$ \\
\hline BMI & & & & & $1.003(0.989-1.018)$ \\
\hline Systolic BP & & & & & $1.012(1.010-1.015)$ \\
\hline Fasting blood glucose & & & & & $1.006(1.005-1.007)$ \\
\hline Total cholesterol & & & & & $1.002(1.001-1.003)$ \\
\hline eGFR & & & & & $0.997(0.995-0.999)$ \\
\hline Smoking amount (pack-year) & & & & & $1.007(1.004-1.010)$ \\
\hline Alcohol intake & & & & & $0.934(0.828-1.053)$ \\
\hline Physical activity & & & & & $0.857(0.764-0.961)$ \\
\hline
\end{tabular}

Notes: *Multivariate adjusted model was adjusted for age, gender, BMI, systolic BP, fasting blood glucose, total cholesterol, eGFR, smoking amount (pack-year), alcohol intake and physical activity.

\section{Abbreviations}

BP, blood pressure; CVD, cardiovascular disease; LDL, lowdensity lipoprotein; BMI, body mass index; AST, aspartate aminotransferase; GGT, $\gamma$-glutamyltransferase; SCr, serum creatinine; eGFR, estimated glomerular filtration rate; CKDEPI, Chronic Kidney Disease Epidemiology Collaboration; NHIS, National Health Insurance Service.

\section{Author Contributions}

All authors made a significant contribution to the work reported, whether that is in the conception, study design, execution, acquisition of data, analysis and interpretation, or in all these areas; took part in drafting, revising or critically reviewing the article; gave final approval of the version to be published; have agreed on the journal to which the article has been submitted; and agree to be accountable for all aspects of the work.

\section{Funding}

This work was supported by a grant from Kyung Hee University in 2019 (KHU- 20191056). This work was supported by the National Research Foundation of Korea in 2020 (grant number: 2020R1G1A1102257). The funding organization had no role in the design or conduct of this study.

\section{Disclosure}

The authors report no conflicts of interest related to this work.

\section{References}

1. Castellano I, Merlino A. $\gamma$-Glutamyltranspeptidases: sequence, structure, biochemical properties, and biotechnological applications. Cell Mol Life Sci. 2012;69(20):3381-3394. doi:10.1007/s00018-012-09883

2. Foyer $\mathrm{CH}$, Noctor G. Redox homeostasis and antioxidant signaling: a metabolic interface between stress perception and physiological responses. Plant Cell. 2005;17(7):1866-1875. doi:10.1105/ tpc. 105.033589

3. Yao T, Li J, Long Q, et al. Association between serum gamma-glutamyl transferase and intracranial arterial calcification in acute ischemic stroke subjects. Sci Rep. 2019;9(1):3-9. doi:10.1038/ s41598-019-56569-7

4. Mason JE, Starke RD, Van Kirk JE. Gamma-glutamyl transferase: a novel cardiovascular risk biomarker. Prev Cardiol. 2010;13 (1):36-41. doi:10.1111/j.1751-7141.2009.00054.x 
5. Ebrahim S, Sung J, Song Y-M, Ferrer RL, Lawlor DA, Davey Smith G. Serum cholesterol, haemorrhagic stroke, ischaemic stroke, and myocardial infarction: Korean national health system prospective cohort study. BMJ. 2006;333(7557):22. doi:10.1136/ bmj.38855.610324.80

6. Sheikh M, Tajdini M, Shafiee A, et al. Association of serum gamma-glutamyltransferase and premature coronary artery disease. Neth Heart J. 2017;25(7-8):439-445. doi:10.1007/s12471-017-0964-5

7. Evans JL, Goldfine ID, Maddux BA, Grodsky GM. Oxidative stress and stress-activated signaling pathways: a unifying hypothesis of type 2 diabetes. Endocr Rev. 2002;23(5):599-622. doi:10.1210/er.2001-0039

8. Zambon A, Pauletto P, Crepaldi G. Review article: the metabolic syndrome-a chronic cardiovascular inflammatory condition. Aliment Pharmacol Ther. 2005;22(Suppl 2):20-23. doi:10.1111/j.13652036.2005.02589.x

9. Lee D-H, Blomhoff R, Jacobs DR. Is serum gamma glutamyltransferase a marker of oxidative stress? Free Radic Res. 2004;38 (6):535-539. doi:10.1080/10715760410001694026

10. Cho YK, Kang YM, Hwang JY, et al. Association between serum gamma-glutamyltransferase and the progression of coronary artery calcification. Atherosclerosis. 2015;243(1):300-306. doi:10.1016/j. atherosclerosis.2015.09.027

11. Jousilahti P, Rastenyte D, Tuomilehto J. Serum gamma-glutamyl transferase, self-reported alcohol drinking, and the risk of stroke. Stroke. 2000;31:1851-1855. doi:10.1161/01.STR.31.8.1851

12. Gurbuzer N, Gozke E, Basturk ZA. Gamma-glutamyl transferase levels in patients with acute ischemic stroke. Cardiovasc Psychiatry Neurol. 2014;2014:10-13. doi:10.1155/2014/170626

13. Lee DS, Evans JC, Robins SJ, et al. Gamma glutamyl transferase and metabolic syndrome, cardiovascular disease, and mortality risk: the Framingham Heart Study. Arterioscler Thromb Vasc Biol. 2007;27 (1):127-133. doi:10.1161/01.ATV.0000251993.20372.40

14. Lee J, Lee JS, Park SH, Shin SA, Kim KW. Cohort profile: the national health insurance service-national sample Cohort (NHIS-NSC), South Korea. Int $J$ Epidemiol. 2017;46(2):e15. doi:10.1093/ije/dyv319

15. Levey AS, Stevens LA, Frcp C, et al. A new equation to estimate glomerular filtration rate. Ann Intern Med. 2009;150(9):604-612. doi:10.7326/0003-4819-150-9-200905050-00006
16. Bots ML, Salonen JT, Elwood PC, et al. $\gamma$-Glutamyltransferase and risk of stroke: the EUROSTROKE project. J Epidemiol Community Health. 2002;56(SUPPL. 1):25-29. doi:10.1136/jech.56.suppl_1.i25

17. Jeon J, Kim DH, Kim W, Choi DW, Jung KJ, Jang SI. Dose-response relationship between gamma-glutamyltransferase and the risk of atherosclerotic cardiovascular diseases in Korean adults. Atherosclerosis. 2020;292:152-159. doi:10.1016/j. atherosclerosis.2019.11.004

18. Yang W, Kim CK, Kim DY, Jeong HG, Lee SH. Gamma-glutamyl transferase predicts future stroke: a Korean nationwide study. Ann Neurol. 2018;83(2):375-386. doi:10.1002/ana.25158

19. Ndrepepa G, Kastrati A. Gamma-glutamyl transferase and cardiovascular disease. Ann Transl Med. 2016;4(24):1-14. doi:10.21037/ atm.2016.12.27

20. Tu W-J, Liu Q, Cao J-L, Zhao S-J, Zeng X-W, Deng A-J. $\gamma$-Glutamyl transferase as a risk factor for all-cause or cardiovascular disease mortality among 5912 ischemic stroke. Stroke. 2017;48 (10):2888-2891. doi:10.1161/STROKEAHA.117.017776

21. Menezes AR, Lavie CJ, Dinicolantonio JJ, et al. Cardiometabolic risk factors and atrial fibrillation. Rev Cardiovasc Med. 2013;14(2-4): e73-81.

22. Ndrepepa G, Xhepa E, Colleran R, et al. Gamma-glutamyl transferase and atrial fibrillation in patients with coronary artery disease. Clin Chim Acta. 2017;465:17-21. doi:10.1016/j.cca.2016.12.003

23. Tekin G, Tekin YK, Senarslan DA, Gocmen AY, Senarslan O, Erbay AR. Serum $\gamma$-glutamyltransferase activity in patients with nonvalvular atrial fibrillation. Angiology. 2013;64(2):157-160. doi: $10.1177 / 0003319712438956$

24. Kunutsor SK, Laukkanen JA, Bluemke DA, Butler J, Khan H. Baseline and long-term gamma-glutamyltransferase, heart failure and cardiac arrhythmias in middle-aged Finnish men: prospective study and pooled analysis of published evidence. Eur J Prev Cardiol. 2016;23(13):1354-1362. doi:10.1177/2047487316644086

25. Alonso A, Misialek JR, Amiin MA, et al. Circulating levels of liver enzymes and incidence of atrial fibrillation: the atherosclerosis risk in communities cohort. Heart. 2014;100(19):1511-1516. doi:10.1136/ heartjnl-2014-305756
Neuropsychiatric Disease and Treatment

\section{Publish your work in this journal}

Neuropsychiatric Disease and Treatment is an international, peerreviewed journal of clinical therapeutics and pharmacology focusing on concise rapid reporting of clinical or pre-clinical studies on a range of neuropsychiatric and neurological disorders. This journal is indexed on PubMed Central, the 'PsycINFO' database and CAS, and

\section{Dovepress}

is the official journal of The International Neuropsychiatric Association (INA). The manuscript management system is completely online and includes a very quick and fair peer-review system which is all easy to use. Visit http://www.dovepress.com/testimonials.php to read real quotes from published authors. 